\title{
Channel nuclear pore protein 54 directs sexual differentiation and neuronal wiring of female reproductive behaviors in Drosophila
}

\author{
Mohanakarthik P. Nallasivan ${ }^{1 \dagger}$, Irmgard U. Haussmann ${ }^{1,2+}$, Alberto Civetta ${ }^{3}$ and Matthias Soller ${ }^{1,4^{*}}$ (D)
}

\begin{abstract}
Background: Female reproductive behaviors and physiology change profoundly after mating. The control of pregnancy-associated changes in physiology and behaviors are largely hard-wired into the brain to guarantee reproductive success, yet the gene expression programs that direct neuronal differentiation and circuit wiring at the end of the sex determination pathway in response to mating are largely unknown. In Drosophila, the post-mating response induced by male-derived sex-peptide in females is a well-established model to elucidate how complex innate behaviors are hard-wired into the brain. Here, we use a genetic approach to further characterize the molecular and cellular architecture of the sex-peptide response in Drosophila females.
\end{abstract}

Results: Screening for mutations that affect the sensitivity to sex-peptide, we identified the channel nuclear pore protein Nup54 gene as an essential component for mediating the sex-peptide response, with viable mutant alleles leading to the inability of laying eggs and reducing receptivity upon sex-peptide exposure. Nup54 directs correct wiring of eight adult brain neurons that express pickpocket and are required for egg-laying, while additional channel Nups also mediate sexual differentiation. Consistent with links of Nups to speciation, the Nup54 promoter is a hot spot for rapid evolution and promoter variants alter nucleo-cytoplasmic shuttling.

Conclusions: These results implicate nuclear pore functionality to neuronal wiring underlying the sex-peptide response and sexual differentiation as a response to sexual conflict arising from male-derived sex-peptide to direct the female post-mating response.

Keywords: Nup54, Nuclear pore complex (NPC), Sexual differentiation, Neuronal wiring, Post-mating behaviors, pickpocket (ppk) neurons

\footnotetext{
* Correspondence: m.soller@bham.ac.uk

${ }^{\dagger}$ Mohanakarthik P. Nallasivan and Irmgard U. Haussmann contributed equally to this work.

${ }^{1}$ School of Biosciences, College of Life and Environmental Sciences, University of Birmingham, Edgbaston, Birmingham B15 2TT, UK

${ }^{4}$ Birmingham Centre for Genome Biology, University of Birmingham, Edgbaston, Birmingham B15 2TT, UK

Full list of author information is available at the end of the article
}

(c) The Author(s). 2021 Open Access This article is licensed under a Creative Commons Attribution 4.0 International License, which permits use, sharing, adaptation, distribution and reproduction in any medium or format, as long as you give appropriate credit to the original author(s) and the source, provide a link to the Creative Commons licence, and indicate if changes were made. The images or other third party material in this article are included in the article's Creative Commons licence, unless indicated otherwise in a credit line to the material. If material is not included in the article's Creative Commons licence and your intended use is not permitted by statutory regulation or exceeds the permitted use, you will need to obtain permission directly from the copyright holder. To view a copy of this licence, visit http://creativecommons.org/licenses/by/4.0/ The Creative Commons Public Domain Dedication waiver (http://creativecommons.org/publicdomain/zero/1.0/) applies to the data made available in this article, unless otherwise stated in a credit line to the data. 


\section{Background}

In most insects, male-derived substances transferred during mating direct female physiology and post-mating behaviors [1, 2]. Drosophila females display a repertoire of sex-specific behaviors after mating including reduced receptivity (readiness to mate) and increased egg-laying [3]. The main trigger of these post-mating behaviors is male-derived sex-peptide (SP). SP is a 36 amino acid peptide that is transferred during mating to the female. Besides reducing receptivity and increasing egg-laying, SP induces a number of other behavioral and physiological changes including increased egg production, feeding, a change in food choice, sleep, memory, constipation, and stimulation of the immune system [411]. In addition, SP binds to the sperm and acts as a sperm sensor, is required for the release of the stored sperm, and imposes costs of mating [12-14].

Many of the phenotypic effects of SP on female physiology are known, but the central aspects of the neuronal circuitry governing the regulation of the main postmating behaviors like reduced receptivity and increased oviposition are unclear. First insights came from the analysis of mutant alleles of the egghead gene, encoding a 1,4 mannosyltransferase involved in glycosphingolipid biosynthesis, that are insensitive to SP and required for neuronal wiring $[15,16]$. In these mutants, neuronal connections from the ventral nerve cord to the central brain show defects resulting in an egg retainer phenotype and non-responsiveness to SP in reducing receptivity. This suggests that receptor signaling is disconnected from the motor output programs.

Other attempts to map the circuitry mediating the post-mating response used expression of membranetethered SP or RNAi knockdown of a receptor for SP, SPR, in distinct neuronal expression patterns [17-21]. These screens identified pickpocket ( $p p k)$, fruitless (fru), and $d s x$ expressing neurons mediating the post-mating switch via expression of membrane-tethered SP in multiple pathways [21].

The ability of males to manipulate post-mating responses of females, for example, by SP in Drosophila, can promote sexual conflict and trigger an arms race between the sexes driven by the female escape of male manipulation [22-24]. This arms race drives rapid evolution and can fuel speciation. Few genes have been linked to speciation by mapping inviability or sterility in hybrids of close relatives. Among them are the two essential nuclear pore proteins, Nup96 and Nup160, that are part of the outer ring of the pore and interact with each other and that can trigger inviability in species hybrids through adaptive divergence [25-28]. The megadalton nuclear pore complex constitutes a bidirectional gateway connecting the nucleus and cytoplasm to control the transport of all macromolecules [29], but how nuclear pore proteins can drive speciation is unknown. Here, we show that Nup54 directs nucleo-cytoplasmic shuttling important for sexual differentiation including wiring of the neurons required for the female post-mating response to SP. Moreover, the Nup54 promoter is a hot spot for rapid diversification consistent with a role in sexual conflict-driven speciation.

\section{Results \\ Nup54 mutant females retain eggs and are insensitive to sex-peptide for reducing receptivity}

If the SP response is disrupted to render females insensitive to SP, they retain eggs and remate, suggesting a common part in the signaling pathway leading to egg-laying and refractoriness to remate upon SP exposure [15]. To identify genes involved in specifying the SP response, we identified lines where females have normal oogenesis, but do not lay eggs. We then tested virgin females of these lines after SP injection for their ability to reduce receptivity [15]. Using this approach, we identified a homozygous viable EMSinduced line, QB62 [30] that did not reduce receptivity upon injection of SP (Fig. 1A-D). We mapped this allele by meiotic recombination based on the egg retention phenotype to 2-69.9, corresponding approximatively to chromosome position 50C. Using overlapping deficiencies this allele could then be mapped to chromosome section 49A4 by deficiencies $D f(2 R) B S C 305$ and $D f(2 R)$ Exel16061 (Fig. 1A). To further restrict the number of genes in this chromosomal area, we generated a smaller deficiency, $D f(2 R) 9 B 4$, by FRT-mediated recombination between transposons $P\{X P\} C G 8525^{d 06853}$ and $P B a c\{R B\} D U$ $B A I^{e 00699}$ (Fig. 1A).

We then identified a GFP-marked $M i\{E T 1\}$ transposon insert in the Nup54 gene, Nup54 ${ }^{M B 03363}$, that was allelic to $Q B 62$ with respect to both egg retention and reduction of receptivity after SP injection (Fig. 1B-D). Nup54 localizes to the transport channel of the megadalton nuclear pore complex that constitutes a bidirectional gateway connecting the nucleus and cytoplasm to control the transport of all macromolecules [29]. The Nup54 $4^{M B 03363}$ transposon insert leads to a truncation of the Nup54 ORF and removes the C-terminal alphahelical domain that connects Nup54 with Nup58, but leaves the core part containing the FG repeats composing the inner nuclear channel and the interaction of Nup54 with Nup62 via the alpha/beta helical region intact (Fig. 1E) [29]. Sequencing of the Nup54 ${ }^{\mathrm{QB6} 2}$ allele identified a small deletion (CATTAGAGAGGAGGCG TAA) in the promoter region $186 \mathrm{nt}$ upstream of the first transcribed nucleotide. These two alleles represent hypomorphic mutations. 


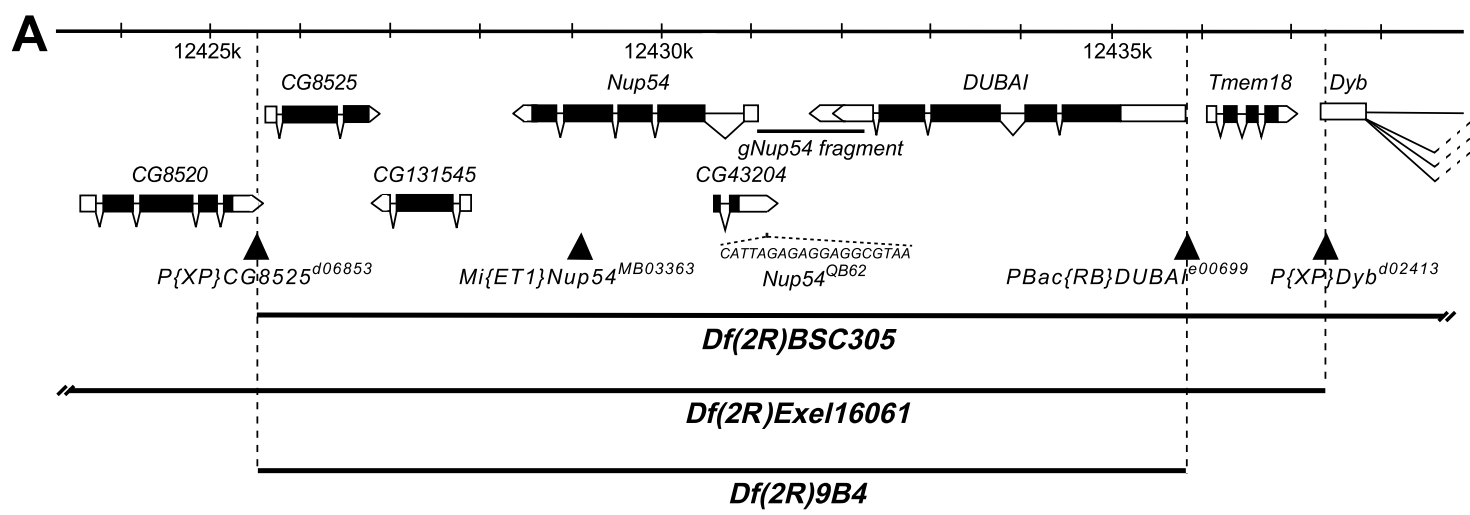

B

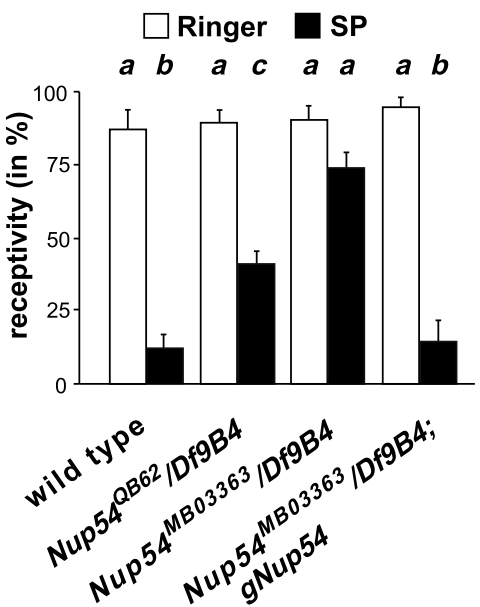

C

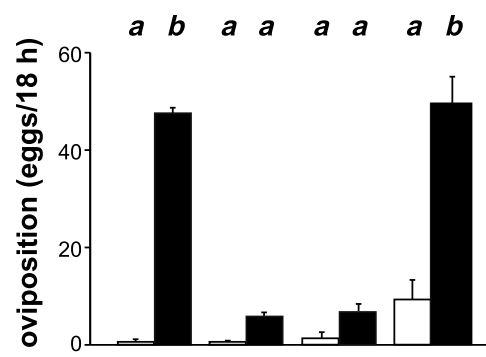

D

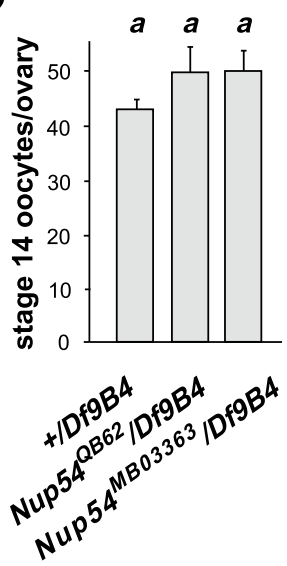

$\mathbf{E}$

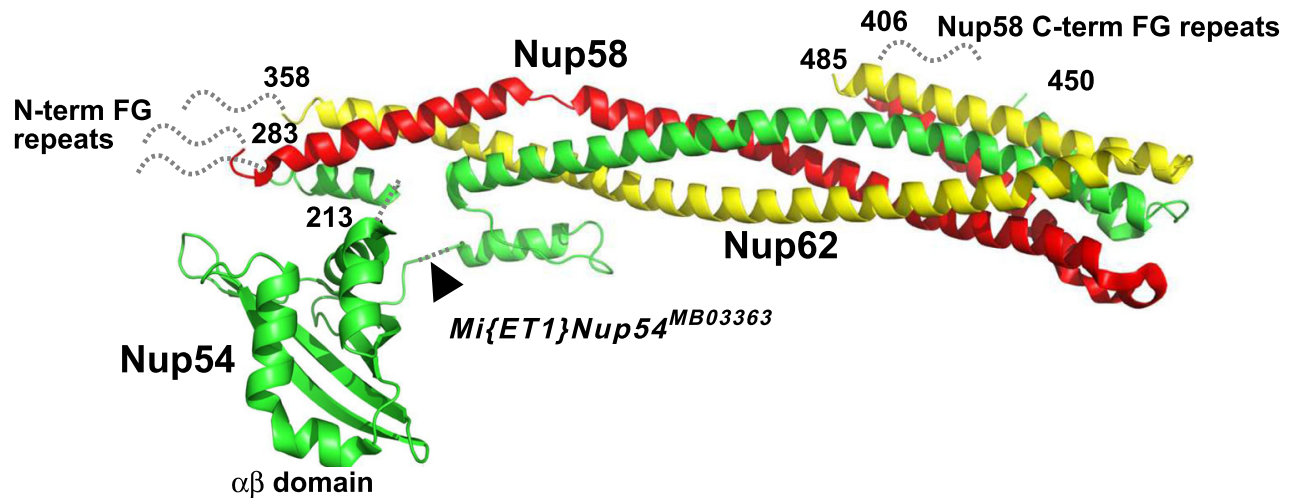

Fig. 1 Mapping of sex-peptide insensitive EMS allele QB62 to Nup54. A Schematic of the Nup54 chromosomal region depicting gene models and chromosomal deficiencies used below the chromosomal nucleotide positions. Coding parts are shown as black and non-coding parts as white boxes. Transposon insertions are show as triangles. The sequence of the deletion in Nup54 ${ }^{\mathrm{B} 62}$, and the promoter fragment used for genomic rescue construct gNup54 are shown below the gene model. B Receptivity of wild type and transheterozygous Nup54 QB62/Df(2R)9B4, Nup54 MBO3363/ Df(2R)9B4, and Nup54 MB03363/Df(2R)9B4; gNup54 females after sex-peptide (SP, black) or Ringer's (R, white) injection measured by counting mating females in a $1 \mathrm{~h}$ time period $3 \mathrm{~h}$ after SP or R injection, respectively. The means with the standard error for three or four experiments with 18-23 females each are shown, and statistically significant differences from ANOVA post hoc pairwise comparisons are indicated by different letters ( $\mathbf{a}, \mathbf{b}$

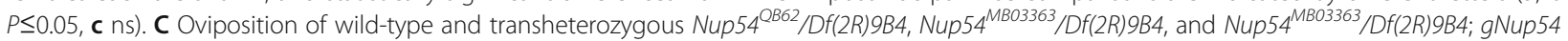
females after sex-peptide (SP, black) or Ringer's ( $\mathrm{R}$, white) injection shown as means of eggs laid in $18 \mathrm{~h}$ with the standard error for 10 females each, respectively, and statistically significant differences from ANOVA post hoc pairwise comparisons are indicated by different letters ( $P \leq 0.0001)$. D Number of stage 14 oocytes present in ovaries of sexually mature virgin wild type and transheterozygous Nup54 $4^{\text {QB62 }} / \mathrm{Df}(2 \mathrm{R}) 9 \mathrm{9B4}$ and Nup54 ${ }^{\mathrm{MBO} 03363}$ /Df(2R)9B4 females shown as means with the standard error for seven ovaries. E Ribbon diagram of the structure of the channel Nup complex consisting of Nup54, Nup58, and Nup62 with the position of the Mi\{ET1\}Nup54 ${ }^{\text {BBO3363 }}$ transposon insertion indicated leading to a partially functional truncated protein. The data underlying the presented graphs are in Additional file 6 
To further confirm that egg retention and SP insensitivity in receptivity maps to the Nup54 gene, we used a genomic rescue construct. For this construct, a fragment upstream of the transcription start site (indicated by a line in Fig. 1A) was fused to the transcribed part, where the first intron containing the overlapping gene CG43204 (gNup54) was deleted. The presence of this construct as in Nup54 $4^{M B 03363} / D f(2 R) 9 B 4 ; g N u p 54$ fully rescued the egg retention and SP insensitivity in receptivity (Fig. 1B, C) and also rescued the lethality of $D f(2 R) 9 B 4$ indicating that Nup54 is essential and the only lethal gene in $D f(2 R) 9 B 4$.

\section{Nup54 is required in the development for establishing the post-mating response}

The SP-induced change in post-mating behaviors is mediated via neuronal signaling [3]. To identify the temporal and spatial requirement of Nup54 during the development of the nervous system, we aimed to rescue the egg retainer and SP insensitivity phenotype in receptivity in Nup54 mutant females by expressing UASNup54 in a Nup5 $4^{M B 03363} / D f(2 R) 9 B 4$ background with various GAL4 drivers. Global expression starting early in development with tubGAL4 in Nup54 $4^{M B 03363}$, $D f(2 R) 9 B 4$ completely rescued these phenotypes after injection of SP (Additional file $1 \mathrm{~A}$ and B). When we then expressed UASNup54 in all differentiated neurons with elavGAL4 ${ }^{C 155}$, refractoriness to remate was not rescued and oviposition increased only marginally in the presence of SP in Nup54 $4^{M B 03363} / D f(2 R) 9 B 4$ (Additional file 1 $\mathrm{A}$ and $\mathrm{B})$. This result indicates that elavGAL4 expresses too late in development to rescue insensitivity to SP to revert irreversible aberrant neuronal development (see below). Compared to the expression of endogenous Nup54, GAL4/UAS-mediated expression is delayed because GAL4 needs to be first made to activate transcription from $U A S$. As a consequence, some gene knockouts such as elav cannot be rescued for this reason [31, 32], but could also be a result of insufficient driver strength in some cells. Expression of UASNup54 in glial cells with repoGAL4 in Nup54 $4^{M B 03363} / D f(2 R) 9 B 4$ rescued receptivity, but not oviposition after SP injection, indicating that the development of the neuronal circuitry mediating the post-mating response is supported by glial cells (Additional file $1 \mathrm{~A}$ and B). Since both elavGal4 and repoGal4 did not fully rescue, it is likely that Nup54 is required in both neurons and glia for the rescue of SP-insensitivity.

We then expressed UASNup54 in those subsets of neurons that can induce the SP response from the expression of membrane-tethered SP using ppkGAL4, fruGAL4, and $d s x G A L 4$ in Nup54 $4^{M B 03363} / D f(2 R) 9 B 4$ after SP injection (Additional file $1 \mathrm{~A}$ and $\mathrm{B}$ ). These experiments revealed that fruGAL4 and $d s x G A L 4$ can rescue refractoriness to remate induced by SP, but only $d s x G A L 4$ can marginally rescue egg-laying. These results indicate that irreversible aberrant neuronal development has taken place before these GAL4 drivers express or that other neurons directing the post-mating response are involved (Additional file $1 \mathrm{~A}$ and $\mathrm{B}$ ).

Taken together, these data show that Nup54 is required early in development before neurons are fully differentiated and in glial cells to specify the neuronal circuits required for the post-mating response as the mutant phenotype can only be rescued by early expressing GAL4 lines. Moreover, our data confirm that the neuronal circuits for egg-laying and receptivity are not identical [21].

\section{Nup54 is required for neuronal wiring of pickpocket neurons}

To evaluate whether Nup54 $4^{\mathrm{QB} 62} / \mathrm{Df}(2 \mathrm{R}) 9 \mathrm{~B} 4$ mutant females had any gross morphological changes in the brain, we analyzed neuronal projections of $p p k$ and $d s x$ neurons as these neurons are directly relevant to female sexual behaviors [17-19, 21] (Fig. 2 and Additional file 2). Moreover, since ppkGAL4 and $d s x G A L 4$ mark only few neurons with distinct axonal tracts as shown with UASCD8GFP expression, they are well suited to evaluate the morphology of relevant neurons (Fig. 2G, H, Additional file $2 \mathrm{~A}$ and $\mathrm{B}$ ).

For $d s x$ neuronal projections in Nup54 $4^{\mathrm{QB62}}$ / $D f(2 R) 9 B 4$ females, we did not detect alterations in the brain $(n=6)$, the ventral nerve cord $(n=6)$, and the genital tract $(n=6)$ compared to wild type (Additional file $2 \mathrm{~A}-\mathrm{F})$. In contrast, projections of $p p k$ neurons in the larval brain of Nup54 $4^{\mathrm{QB} 62} / D f(2 R) 9 B 4$ showed severe disruption with connectives and/or commissures in $50 \%(n=20)$ of the brains, and moderate and mild aberrations in half of the remaining brains each compared to wild type (Fig. 2A-F). In the adult brain, projections in the central brain were severely aberrant in $89 \%$ of $N u p 54^{Q B 62} / D f(2 R) 9 B 4$ females $(n=9)$ and mildly aberrant in the remaining brains compared to control females ( $n=10$, Fig. 2G, H), while no projection differences were obvious in the ventral nerve cord ( $n=5$, Fig. 2I, J). A closer examination of ppkGAL4 expression with nuclear UAShistone2B::YFP, however, revealed four paired neurons that are consistently present in the adult female brains $(n=5$, Fig. $2 \mathrm{~K})$ [33].

Most prominently, $p p k$ expresses in sensory neurons projecting into the brain [17-19], but their contribution to arborizations of $p p k$ neurons in the brain has not been established. Since ascending neurons from the genital tract and the ventral nerve cord project to the brain, we used flipase ( $f l p$ ) expression under an orthodenticle (otd) promoter [34], which is restricted to the 

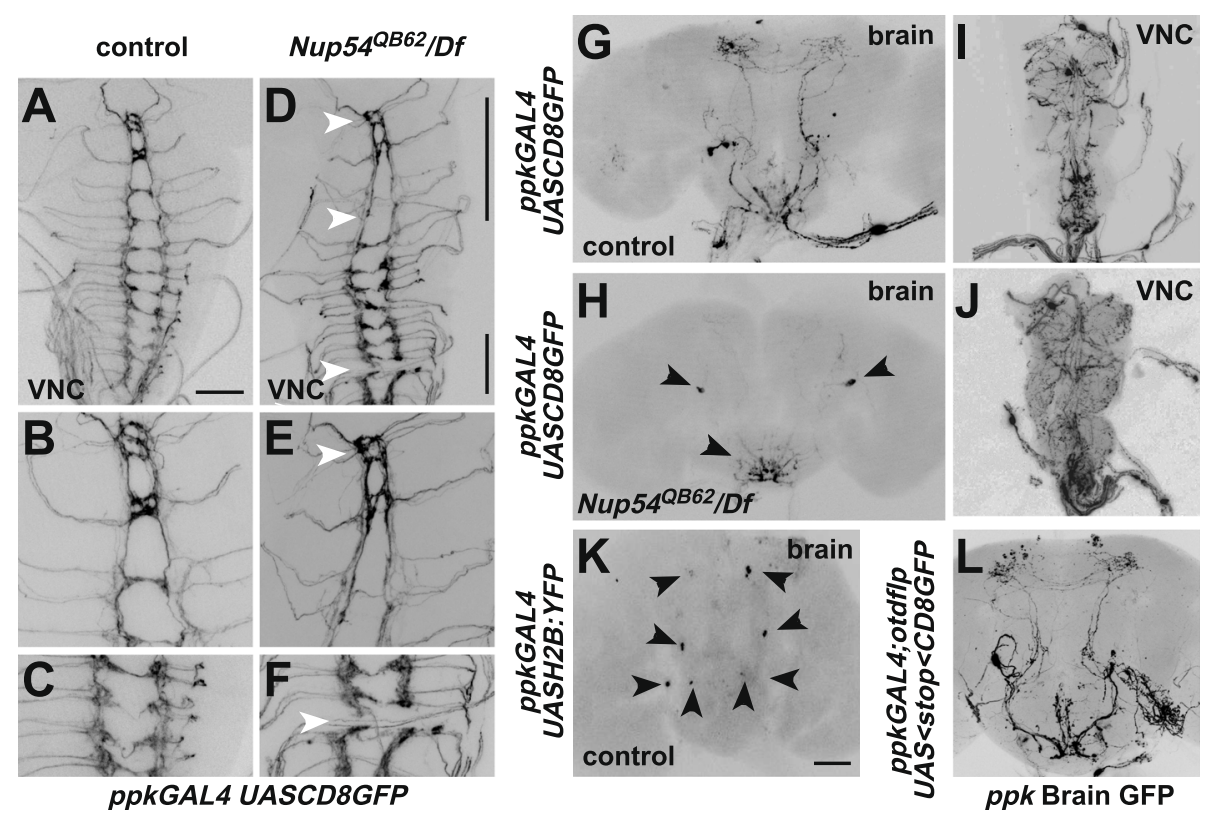

Fig. 2 Nup54 is required to establish neuronal projections of pickpocket neurons. A-F Representative larval ventral nerve cord expressing CD8::GFP from UAS by ppkGAL4 in wild type (A-C) and Nup54 ${ }^{\mathrm{QB} 62} / \mathrm{Df}(2 R) 9 B 4$ (D-F). Magnified corresponding areas indicated in $\mathbf{D}$ are shown for wild-type (B and $\mathbf{C}$ ) and Nup54 ${ }^{\mathrm{QB62}} / \mathrm{Df}(2 R) 9 B 4$ (E and $\mathbf{F}$ ). Arrowheads indicate missing commissures or connectives. G-J Representative adult brain and ventral nerve cord (VNC) expressing CD8::GFP from UAS by ppkGAL4 in wild type (G and $\mathbf{I})$ and Nup54 $4^{\mathrm{QB} 62} / \mathrm{Df}(2 R) 9 B 4$ (H and $\left.\mathbf{J}\right)$. Arrowheads point towards cell bodies of ppk expressing neurons with absent neuronal arborizations. K Representative adult brain expressing histone2B::YFP from UAS by ppkGAL4 in wild-type. Arrowheads point towards cell bodies of the four paired ppk expressing neurons in the adult brain. The scale bar in $\mathbf{A}$ is $50 \mu \mathrm{m}$ and in $\mathbf{K}$ is $100 \mu \mathrm{m}$. L Representative adult brain expressing CD8::GFP only in head ppk neurons by the intersection of ppkGAL4/UAS FRTstopFRT CD8GFP expression with otdflp, which expresses only in the brain

brain to determine whether the arborizations in the brain are from these four $p p k$ neuron-pairs or result from projections from neurons in the genital tract. No difference in the projections in the central brain was observed when $p p k$ expression was restricted to the brain ( $n=4$, Fig. 2L) with an intersectional approach (Fig. 3A). Accordingly, GFP expression was absent in genital tract $p p k$ neurons in these females $(n=4)$ as otd-flp is only expressed in the head and therefore does not remove the stop cassette in UASCD8FRTstopFRTGFP in the genital tract (Fig. 3B, C). In Nup54 $4^{\mathrm{QB62}} / D f(2 R) 9 B 4$ genital tracts, ppk neurons were present and showed no major arborization defects or disruption in the projections $(n=$ 4, Fig. 3D).

\section{ppk neurons in the brain direct egg-laying}

Since expression of membrane-tethered SP with $p p k G A L 4$ induces a post-mating response, we wanted to test whether $p p k$ expressing neurons in the brain are involved in the post-mating response. For this experiment, we used the same intersectional gene expression approach using otd-flp to specifically inhibit neuronal transmission in brain $p p k$ neurons by expression of tetanus toxin (TNT) (Fig. 3A). To achieve restricted expression of TNT in ppk brain neurons, we crossed otdflp flies with ppkGAL4; UAS FRTGFPstopFRT TNT flies and analyzed the female progeny for the post-mating response.

These experiments revealed that females with inhibited $p p k$ neurons in the brain or with all $p p k$ neurons inhibited displayed a partial response to SP as they did not fully reduce receptivity (Fig. 3E). Females with inhibited $p p k$ neurons in the brain did not lay the eggs stored in the ovaries after injection of SP in contrast to inhibition of all $p p k$ neurons which resulted in increased egglaying in virgins and SP does not further enhance egglaying (Fig. 3G, F). Control females of UAS TNT without a GAL4 driver showed a normal response to SP [15].

Taken together, these results demonstrate an essential role for $p p k$ neurons in the brain in inducing egg-laying, and a partial requirement for reducing receptivity in response to SP, but also that $p p k$ neurons outside the brain are inhibitory with regard to egg-laying as reported previously [17-19].

\section{Channel Nups have a role in sex determination}

To further examine the role of Nup54 in the postmating response, we used UASRNAi knock-down of Nup54 ubiquitously with tubGAL4, in all neurons using elavGAL4 ${ }^{C 155}$, or in subsets of neurons using ppkGAL4, fruGAL4, and dsxGAL4. RNAi knock-down of Nup54 with tubGAL4 is lethal, while elavGAL4 ${ }^{C 155}$, ppkGAL4, 


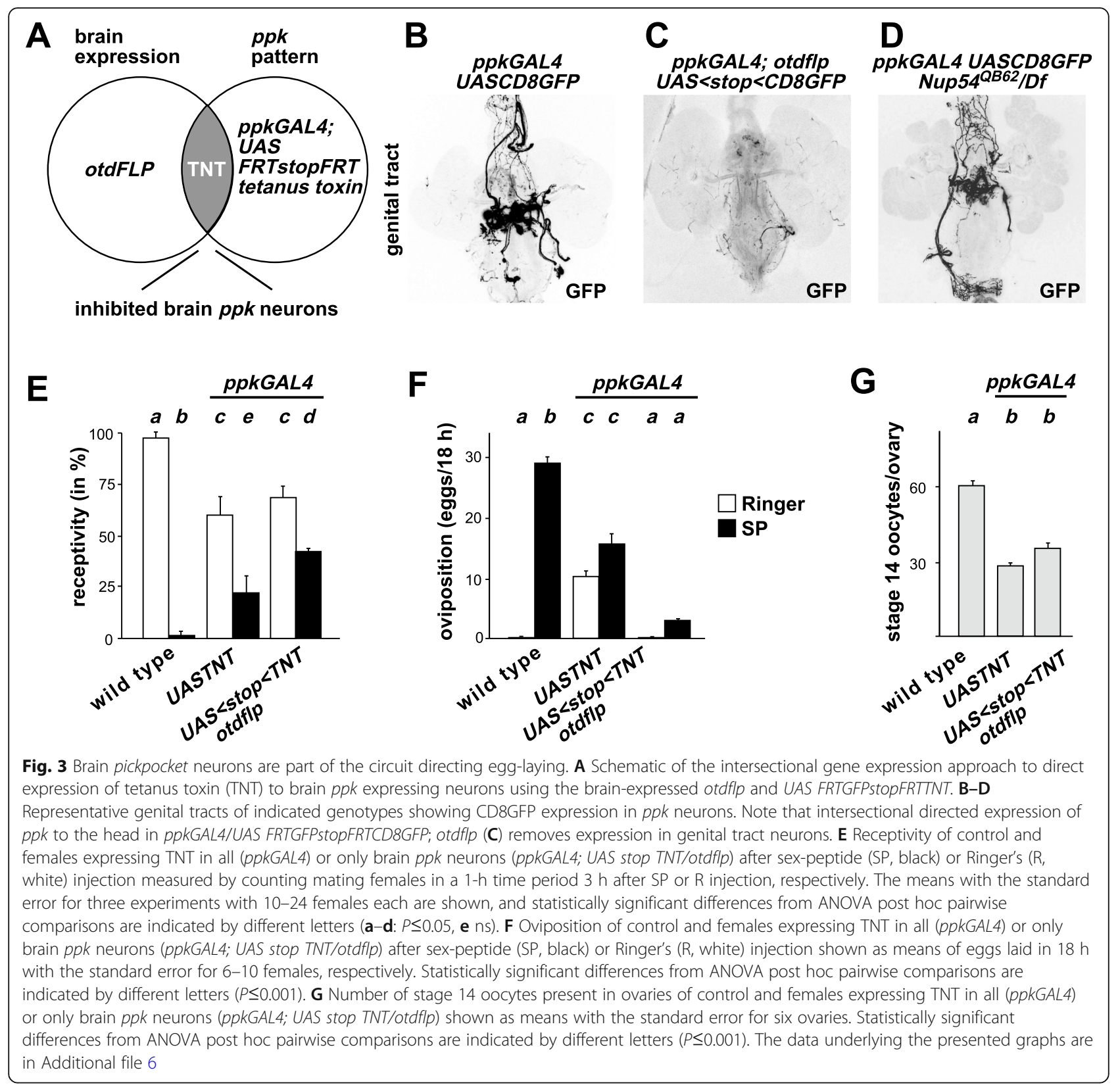

and fruGAL4 did not affect receptivity, or egg-laying after SP injection (Additional file $3 \mathrm{~A}$ and $\mathrm{B}$ ). This result is not unexpected since Nup54 is required early in neuronal development to rescue SP insensitivity in Nup54 mutant females, as shown by the lack of rescue from GAL4 lines expressing a rescue construct late in development, but alternatively, RNAi knock-down could insufficiently reduce Nup54 expression. In contrast, RNAi knock-down of Nup54 with $d s x G A L 4$, oviposition was not induced upon SP injection while receptivity was reduced normally (Additional file $3 \mathrm{~A}$ and B).

We further noticed that these dsx Nup54 RNAi females laid eggs that did not develop. Moreover, male survival was low $(20 \%, n=107, P\{$ TRIP.HMC04733 $\})$ or males were completely absent (n=107, $P\{G D 14041\} v 42153 ; P\{G D 14041\} v 42154)$. In the progeny of the mild hypomorphic Nup54 $4^{\mathrm{QB62}}$ and Nup5 $4^{M B O 3363}$ alleles crossed to $D f(2 R) 9 B 4$, no bias in male survival was detected (98\% males, $n=194$ and $92 \%$ males, $n=142$, respectively).

Since Nup54 forms a complex with two other channel Nups, Nup58 and Nup62, we wanted to test by RNAi whether they have roles in specifying the post-mating response and/or in sexual differentiation. RNAi knockdown of Nup58 (P\{TRIP.HMC05104\}) with dsxGAL4 resulted in lethality with only few very weak female 
escapers. These females, however, were sterile due to the lack of ovaries and deformed genitals, but looked otherwise normal. Similar results were obtained by RNAi knock-down of Nup62 with $d s x G A L 4$ resulting in male lethality (9\%, $n=81$ and $2 \%, n=45$ escapers with $P\{$ TRIP.GLV21060 $\}$ and P\{TRIP.HMC03668\}, respectively) and females without ovaries (100\%, $n=74$ and $43 \%$, $n=35$ with P\{TRIP.GLV21060 $\}$ and P\{TRIP.HMC03668 $\}$, respectively). In addition, these females also had deformed genitals and males displayed underdeveloped sex combs indicating a role for channel Nups in sexual differentiation (Additional file $3 \mathrm{C}-\mathrm{F}$ ). Consistent with a regulatory role in development, the channel Nups display dynamic expression during development and in different tissues (Additional file $4 \mathrm{~A}$ and B) $[35,36]$.

\section{The role of indels during the evolution of Nup54 function} Changes in the protein sequence of Nups have been attributed a role in speciation as an adaptation to new situations drives the evolution of Nup96 and Nup160 genes by leading to hybrid incompatibility [25-27]. Therefore, we tested whether the Nup54 coding region is under selection. We used the combined analysis of polymorphism within $D$. melanogaster and divergence between $D$. melanogaster and D. simulans within the coding region of Nup54 to test for deviations from expectations under the neutral model of molecular evolution. We found no deviation from the null neutral model hypothesis, as the ratio of nonsynonymous to synonymous polymorphisms did not differ significantly from the ratio of nonsynonymous to synonymous fixed differences between species (MK test: $\chi^{2}=0.09 ; P=$ $0.923)$. The test was also non-significant when we partition the analysis by exons.

When we compared the sequences in more distantly related species, we noticed that a stretch of amino acids increased in length inserted in the FG repeat region constituting the transport channel (Fig 1E and Additional file $5 \mathrm{~A}$ ). Indels (insertions/deletions) are a common form of genetic variation that can affect gene function and their pattern of evolution under selective forces [37]. We therefore hypothesized that this indel could alter the function of Nup54 in specifying the postmating response.

To test this hypothesis, we replaced the FG-repeat region from $D$. melanogaster with the region from $D$. elegans in the genomic rescue construct gNup54ele and tested its capacity to rescue viability of $D f(2 R) 9 B 4$ and the post-mating response defects. Both the $D$. melanogaster gNup54 and the chimeric D. elegans construct $g N u$ p54ele rescued viability of $D f(2 R) 9 B 4$ to $91 \%(n=242)$ and $95 \%(n=381)$ without sex bias, respectively. After injection of SP, females rescued with the gNup54 or the gNup54ele construct showed normal post-mating responses in receptivity and oviposition arguing that this indel is not the source for an altered post-mating response (Additional file $5 \mathrm{~B}$ and $\mathrm{C}$ ).

Since the coding region of Nup54 is not under selection, we next examined the Nup54 promoter region. Sequence analysis in this region identified rapid evolution in four hotspots for substitutions between closely related species, all within $1 \mathrm{~kb}$ upstream of the Nup54 transcription start site (Fig. 4A). Interestingly, one substitutional hotspot $(-359$ to -295$)$ includes an indel that overlaps with the location of the characterized Nup54 ${ }^{Q B 62}$ regulatory allele (Fig. 4B). The deletion in the Nup $54^{\mathrm{QB} 62}$ allele in fact is to a large extent a wild-type condition in sister species (Fig. 4A).

To evaluate whether the QB62 allele of Nup54 affects nucleo-cytoplasmic shuttling, we stained brains for ELAV, an RNA-binding protein that shuttles between the nucleus and cytoplasm [32]. While in the brains of wild-type flies, ELAV is mostly nuclear, it is more cytoplasmic in Nup54 $4^{\mathrm{QB6}} / \mathrm{Df}(2 \mathrm{R}) 9 \mathrm{~B} 4$ brains (Fig. 5A-L). Analysis of ELAV's cellular distribution specifically in these central brain $p p k$-expressing neurons with aberrant projections revealed that ELAV was distributed equally in nucleus and cytoplasm (Fig. 5H-L) compared to strong nuclear localization in wild-type $p p k$-expressing neurons, but whether nucleo-cytoplasmic shuttling is altered in species with QB62-like promoters remains to be tested (Fig. 5B-F).

\section{Discussion}

Here, we show that Nup54, which is part of the central transport channel of the nuclear pore, exerts a role in specifying the neuronal circuits involved in mediating the SP-induced post-mating switch. Viable alleles of these genes display an egg retainer phenotype and are insensitive to SP with regard to reducing receptivity. Nup54 is required for wiring of $p p k$ expressing neurons in the brain that constitute part of the circuitry required for egg-laying. The role of Nup54 and the other twochannel Nups, Nup58 and Nup62, in sexual differentiation, however, seems to be more fundamental as reducing their expression levels impacts on general sexual differentiation of external and internal sexual features, such as female genitals and ovaries.

\section{Roles for Nups in development}

A key role for Nups in sexual differentiation seems rather unexpected as the function of channel Nups in directing constitutive export of mRNAs and proteins from the nucleus to the cytoplasm has been viewed as static for a long time. However, a number of recent studies have shown developmental and neuronal roles for the nuclear pore and nucleocytoplasmic transport reflected in a number of human diseases including 


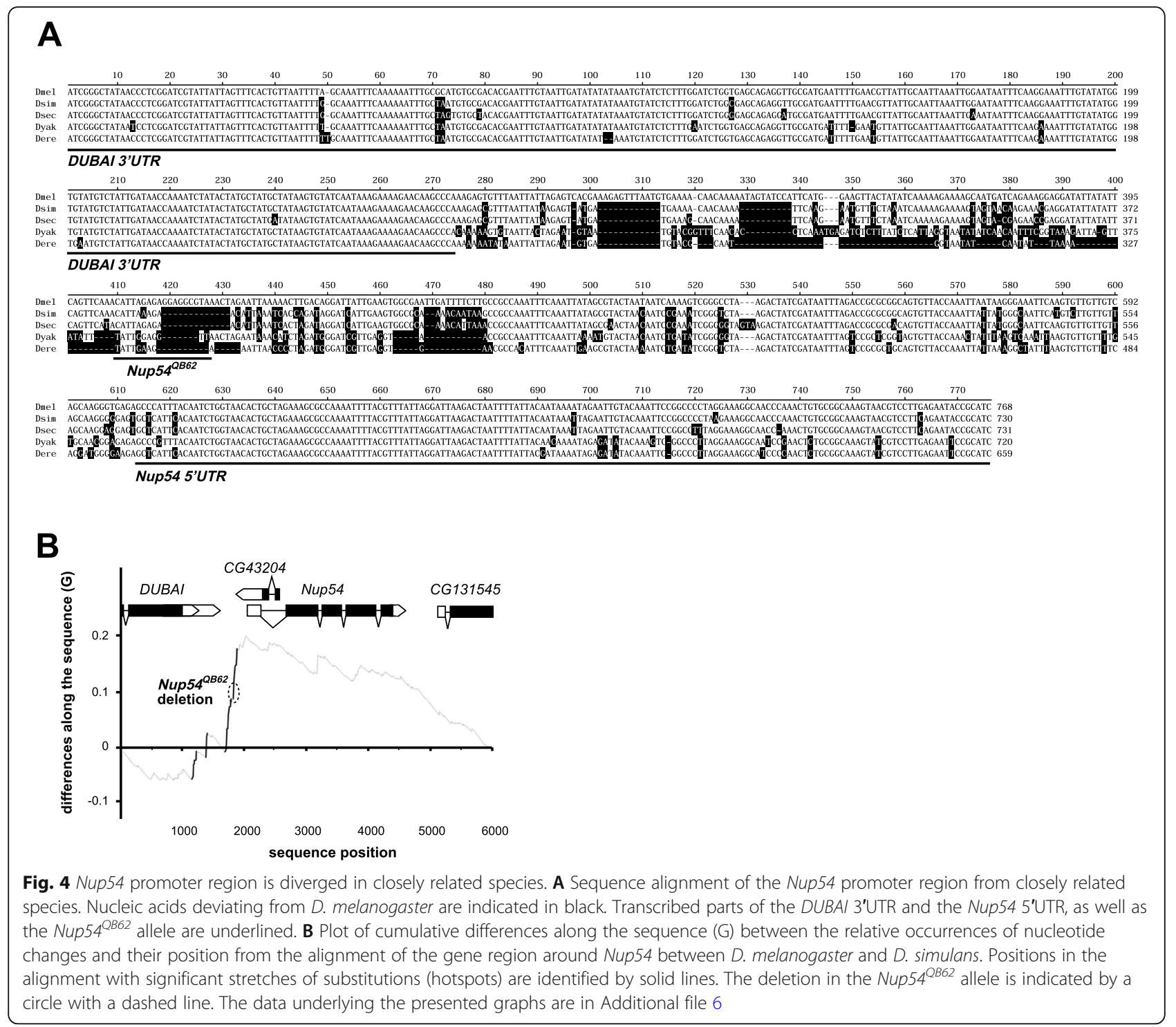

neurodegeneration associated with specific Nups [3840]. A central aspect of these more profound roles for Nups lay in their varying expression levels between different cell types, tissues, and in development. Nup210's key role in muscle and neuronal differentiation is associated with tissue-specific expression [41]. Likewise, high expression levels of Nup153 are critical for the maintenance of ESC pluripotency as reducing levels results in neuronal differentiation [42]. Also, channel Nup62 shows increased expression in various epithelia and is required for proliferation [43]. In Drosophila, channel Nups 54, 58, and 62 show increased expression in the larval brain and adult gonads, but a more specific role is indicated in sexual development in Drosophila from broad RNAi knock-down with $d s x G A L$, recapitulating that $d s x$ expression cell-autonomously directs sex determination $[44,45]$. Our findings that channel Nups have a role in sexual differentiation add to this view that the nuclear pore has key roles in differentiation and likely also neuronal function.

We observed that RNAi of Nup54 and Nup62 results in male lethality. We did not see a sex bias in the mild hypomorphic alleles of Nup54 likely because they retain considerable functionality. In contrast, RNAi of Nup58 resulted in general lethality. Differences among channel Nups have also been seen in piRNA biogenesis of the flamenco locus [46]. Even though channel Nups form a complex, they could have individual roles in nucleocytoplasmic shuttling pathways, but alternatively, phenotypic differences could also be a result of the different strengths of the RNAi lines. Saturation screens for malelethality revealed that all identified male-lethal mutants mapped to genes regulating dosage compensation [32, $44,45]$, but alternative scenarios are possible involving a 

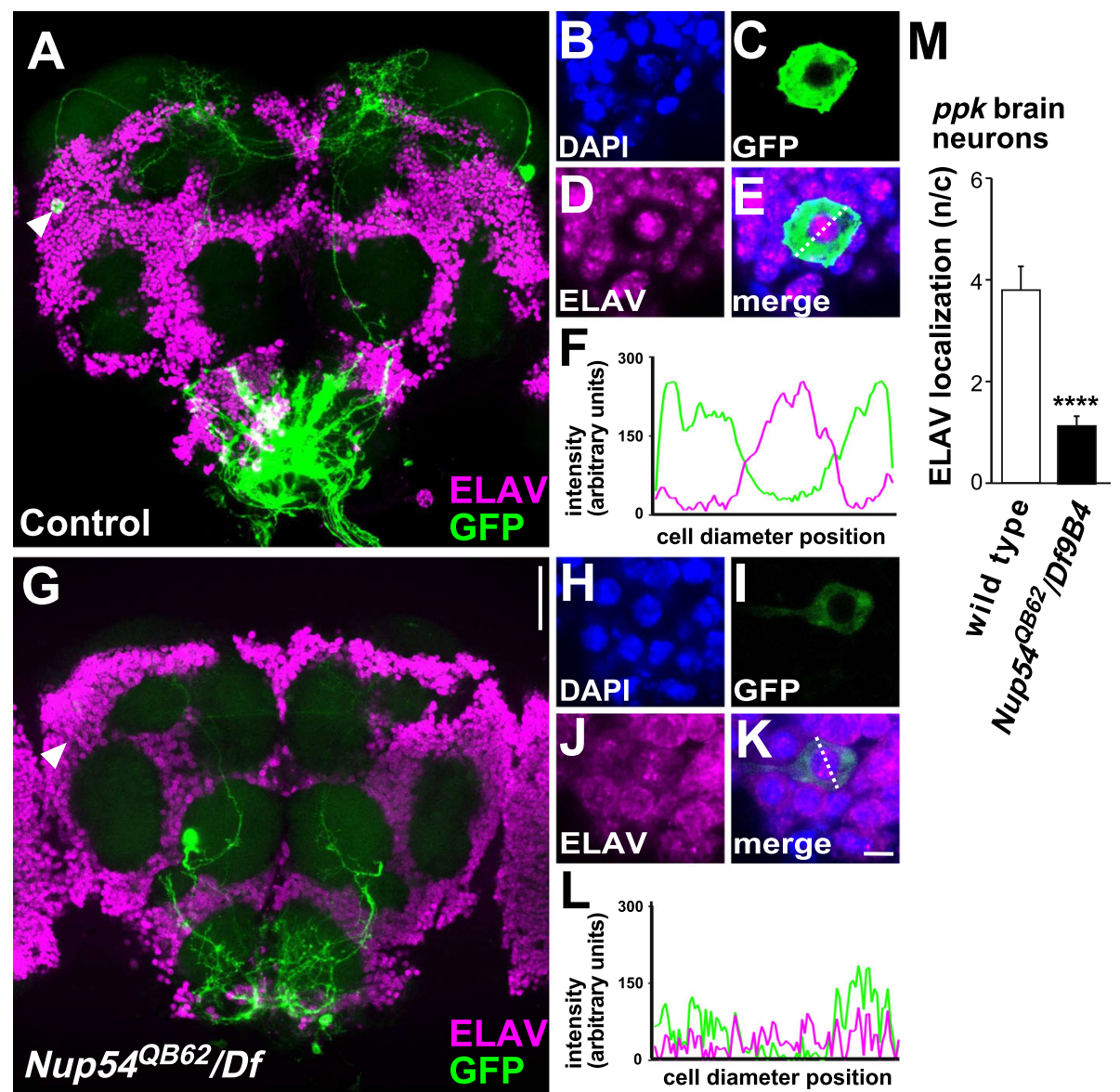

Fig. 5 Nucleo-cytoplasmic shuttling is altered in Nup54 ${ }^{\mathrm{QB62}}$. A-M Cellular localization of nucleo-cytoplasmic shuttling RNA-binding protein ELAV (magenta) in representative control (A-F) and Nup54 ${ }^{\mathrm{QB62}} / \mathrm{Df}(2 R) 9 B 4(\mathbf{G}-\mathbf{L})$ expressing UASCD8GFP from ppkGAL4 (green). Arrowheads in $\mathbf{A}$ and $\mathbf{G}$ indicate the ppk expressing neuron in higher magnification for the control $(\mathbf{B}-\mathbf{E})$ and Nup54 ${ }^{\mathrm{B} 62} / \mathrm{Df}(2 \mathrm{R}) 9 B 4(\mathbf{G}-\mathbf{K})$ and the line in $\mathbf{E}$ and $\mathbf{K}$ indicate the position where signal intensities (arbitrary units) were measured for GFP (green) and ELAV (magenta) plotted in $\mathbf{F}$ and $\mathbf{L}$ of a representative

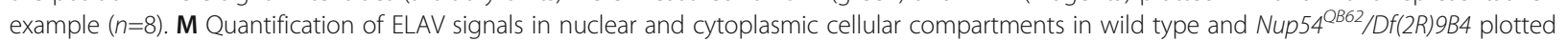
as mean with the standard error of the ratio of the nuclear to the cytoplasmic signal $(P \leq 0.0001)$. The scale bar in $\mathbf{G}$ is $50 \mu \mathrm{m}$ and in $\mathbf{K}$ is $1 \mu \mathrm{m}$. The data underlying the presented graphs are in Additional file 6

stronger role of channel Nups in male than female sexual differentiation. Moreover, MSL-2 expression in females is further inhibited by nuclear retention of $m s l-2$ mRNA by SXL and HOW RNA-binding proteins [47], but whether Nup54 and Nup62 have indeed a role in this process requires further validation.

\section{Specification of neuronal circuits for receptivity and egg- laying are separable}

It has previously been argued that the neurons coexpressing fru, $d s x$, and $p p k$ in the genital tract sense SP and direct the SP-induced post-mating response [1719]. However, our data from expressing a Nup54 rescue construct in various cell types suggests a more complex picture including separation of the paths leading to reduced receptivity and increased egg-laying. Here, the expression of Nup54 in fru and $d s x$ neurons, but not ppk neurons rescues receptivity while egg-laying is not rescued in all three expression patterns. Although this effect could be explained by different expression levels of fru, $d s x$, and ppk GAL4 lines, this is not observed and they all express strongly. Consistent with a requirement for Nup54 early in development, $d s x$ is expressed earlier than $f r u$ and $p p k$. Differential effects on receptivity, but not egg-laying by $d s x$ driven rescue by Nup 54 , however, more likely indicates that receptivity and egg-laying are governed by different neuronal circuits. These results are supported by previous observations that $\mathrm{G}(\mathrm{o})$ is not required in $f r u$, but needed in $d s x$ and $p p k$ neurons to reduce receptivity [21]. Likewise, membrane-tethered SP can only induce oviposition in the absence of SPR in $d s x$, but not fru and ppk neurons [21]. 


\section{Roles of Nups in speciation}

New species can arise through the selection of new features enhancing the display of sexual attributes and altered courtship behavior. A driving force to speciation can be sexual conflict imposed by male-directed postmating responses by females [22-24]. In D. melanogaster, females respond to male-derived SP by reducing receptivity and increasing egg-laying, but need to adapt their physiological status to environmental conditions. Thus, the possibility to limit the male influence imposed by $\mathrm{SP}$ is in the female interest of optimizing reproductive success when resources to produce eggs are scarce. In this context, Nup54 regulation could impact on egglaying in dedicated circuits such as the brain ppk neurons. Consistent with this interpretation, the Nup54 promoter region has undergone a dramatic change compared to closely related species, while no adaptive changes were detected in the protein-coding part. The QB62 deletion in the promoter region of Nup54 affected the ELAV distribution of ppk-expressing neurons and such deletion mimics the wild-type allele condition in sister species of $D$. melanogaster. While we did not perform a direct interspecies allele swap, our result suggests that differences in regulation of Nup54 might be critical in mediating interspecies differences in female reproductive behavior. How allelic variants of Nups lead to reproductive advantages, however, needs to be further explored, but could involve subtle changes in neuronal wiring that alters behavioral preference. In addition, since Nup54 is required in all cells, varying its concentration could have profound effects on the general expression of genes and select for a specific compensatory genetic element from the mating partner to prevent deleterious effects on the fitness of the progeny [25-27], but whether nucleo-cytoplasmic shuttling is affected in species with a QB62-like promoter remains to be tested. The regulation of gene expression at the nuclear pore has been linked to speciation, because essential Nup 96 and Nup160 have been identified in causing hybrid sterility or lethality. Intriguingly, Nup96 and Nup160 are part of the larger Nup107 subcomplex and four out of eight proteins from this complex are under adaptive selection in D. simulans [27].

Nup54 has also been found to interact with the piRNA pathway and interspecific hybrids phenocopy piRNA pathway mutants [48-50]. The piRNA pathway is involved in suppressing the mobilization of transposons in the germline. PIWI further interacts with ELYS at nuclear pores indicating that RNA export through nuclear pores is critical for transposon suppression [51, 52]. In particular, channel Nups are required for piRNA biogenesis of the flamenco locus and through transposon silencing can impact on fecundity [46]. The driving forces behind the role of Nups in speciation clearly seem to go beyond hybrid sterility or lethality. Hence, the molecular mechanism leading to speciation through Nups needs to be further explored.

\section{Conclusions}

We have identified eight $p p k$-expressing neurons in the brain as a cellular focus preventing female escape from male manipulation. Changes in neuronal wiring likely directed in response to sexual conflict arising from malederived SP to direct the female post-mating response marks an early event in the splitting of species and links differentiation of key neurons involved in female control of the reproduction to fitness as a result of sexual conflict. Our results indicate a central role for the nuclear pore in implementing alterations of gene expression impacting on neuronal wiring, which might have been critical at the onset of speciation.

\section{Methods}

Flies were kept on standard cornmeal-agar food (1\%industrial-grade agar, 2.1\% dried yeast, $8.6 \%$ dextrose, 9.7\% cornmeal, and $0.25 \%$ Nipagin, all in $(\mathrm{w} / \mathrm{v}))$ in a 12 -h light: 12 -h dark cycle. Sexually mature 3-5-day-old virgin females were injected and examined for their postmating behaviors non-blinded as described previously [4, 15]. For injections, virgin female flies were cooled to $4^{\circ} \mathrm{C}$ and 3 pmol SP in $50 \mathrm{nl}$ Ringer's solution was injected. The ovaries were analyzed as previously described [4].

In a genetic EMS screen for oogenesis mutants, one class was identified that had normal oogenesis, but females did not lay eggs [30]. To test whether in this class of mutants, we can identify ones that are insensitive to $\mathrm{SP}$, we first validated that homozygous females did not increase egg-laying upon SP injection. Then, we tested whether homozygous females injected with SP reduce receptivity.

For the analysis of larval and adult axonal projection expressing green fluorescent protein (GFP), tissues were dissected in PBS, fixed in 4\% paraformaldehyde in PBS for $15 \mathrm{~min}$, washed in PBST (PBS with BSA and 0.3\% Triton-x 100). Aberrant projections in the larval ventral nerve cord were classified as mild, if there were slight mis-projections in the anterior region, as moderate, if connectives or commissures were thinned indicative of absent projections and as severe, if one or more connectives or commissures were completely disrupted.

Aberrant projections in the adult brain were classified as mild, if there were slight mis-projections, as moderate, if some projections did not innervate the target area and as severe, if entire projections were missing.

In situ antibody stainings were done as described previously [31] using rat anti-HA (MAb 3F10, 1:20; Roche) and mouse anti-ELAV (MAb 7D, 1:20, which recognizes 7 amino acids unique to ELAV) [53] and visualization 
with Alexa Fluor 488 or Alexa Fluor 647-coupled secondary antibodies (1:250; Molecular Probes or Invitrogen, A11034). DAPI (4',6-diamidino-2-phenylindole) was used at $1 \mu \mathrm{g} / \mathrm{ml}$. For imaging, the tissues were mounted in Vectashield (Vector Labs) and visualized with confocal microscopy using a Leica TCS SP8. Images were processed using Fiji. Pictures of fly parts were taken with a Zeiss Stemi200-CS and Zen Blue software. Since egglaying and receptivity data are skewed, we used nonparametric Welch's ANOVA followed by planned pairwise comparisons with Dunnett's multiple comparison test done for statistical analysis using Graphpad prism.

The UAS construct with a N-terminally HA-tagged Nup54 was generated in a three-way ligation with fragments of Nup54 amplified from cDNA by RT-PCR that were cloned into a modified $p U C$, pUC 3GLA UAS HA, with NheI and Acc65I with a blunt site in between. The 5' part was amplified using primers 8831F1 (CATCGC TAGCGCCTGCAGGATCGTTCTTCGGATCCAACAC GTCGCTGG) and 8831R1 (CTGAGAGTTCTCTG CAGAAGTTAAGAGCCAC), and the 3' part with primers 8831F2 (CTGTCAAGCCACACCAGCAA CAAGTGATTC) and 8831R2 (GACAGGTACCTATC ACGATTGTCGCAGCTCGGGCAGTC) by PCR with Pwo (Roche) and sequenced. The genomic rescue construct was generated in a three-way ligation by cloning the promoter fragment amplified from genomic DNA with primers 8831F1g (GTGGAATTCCGGAGGCCA CTAGAACATATACTTGTC) and 8831R1g (GGCGTG CTTGTTGCTCCCAGCGACGTGTTG) and the cDNA part amplified from the $U A S$ construct with primers 8831F3 (GGCCAAAACAACCGGTGGCCTCTTCGGA TC) and 8831R3 (GGCGTGCTTGTTGCTCCCAGCG ACGTGTTGTAGCTCGAGGATTGTCGCAGCT

CGGGCAGTC) using EcoRI and XhoI sites into a modified $p U C, p U C$ 3GLA that adds a C-terminal HA tag and sequenced [54]. Swapping the Nup54 wild type promoter was done by digesting the parent plasmid with BspEI and NgoMIV to insert the Nup54 $4^{\mathrm{QB62}}$ promoter or the promoter from Drosophila simulans (S-23, Ethiopie 225, Welo Ataye River, F. Leumeunier, gift from S. Collier Cambridge) from a PCR amplified with primers 8831F3BspE (GCTTAGGATCCGATCGCGTGGAAT TCCGGAGGCCACTAGAACATATACTTGTC) and 8831promR (CGAAGAGGCCACCGGTTGTTTTGG CCGGCGTGCTTGTTGCTCCCAGCGACGGTTG).

The resulting pUC $3 G L A$ gNup $54^{\mathrm{QB62}}$ and pUC $3 G L A$ gNup $54^{\text {Dsim }}$ constructs were validated by sequencing with primer 8831promR2seq (GGCTGGTTGCAGCT GTGCCTCCAAAC). The D. elegans rescue construct was generated in a three-way ligation by replacing the part between NgoMIV and MfeI with the corresponding part amplified from $D$. elegans cDNA by RT-PCR with primers 8831Fele (CCAAAACAACCGGAGGCCTCTT
CGGAAC) and 8831R1ele (CGTGGATCCGAAGGCT CCGCCCCCAAAGCCAGTG), and a BamHI/MfeI fragment obtained from the UAS construct. Transgenic flies were generated by phiC31-mediated transformation using landing site 76A (PBac\{y+ -attP-3B\}VK00002), where the GFP marker had been removed by Cre/Loxmediated recombination. $D f(2 R) 9 B 4$ was generated by FLP/FRT-mediated recombination between the two transposon insertion lines, $P\{X P\} C G 8525^{d 06853}$ and $P B a c\{R B\} D U B A I^{e 00699}$ as described [32]. The tubGAL4 line was a third chromosomal insert (Bloomington \#5138), and UAS FRTGFPstopFRT TNT and the other lines have been previously described $[19,21,31]$.

Genomic DNA was extracted from Nup $54^{\mathrm{QB} 62}$ as described [55]. The promoter fragment was amplified with primers 8831pF1 (GGATCTGGTGAGCAGAGGTT GCGATG) and 8831pR1 (GCCGCACAGTTTGGGTTG CCTTTC) and sequenced. Reverse transcriptionquantitative polymerase chain reaction (RT-qPCR) was done by extracting total RNA using Tri-reagent (SIGMA), and RT was done with Superscript II (Invitrogen) according to the manufacturer's instructions using an oligo dT primer. For qPCR, $1.5 \mu \mathrm{l}$ cDNA with the SensiFAST SYBR No-Rox kit (Bioline) was used with primers Nup54qF1 (CTGCCACAGCGAAGATACT) and Nup54qR1 (CAGCATGTTCTGTAGCTTGGTGC), and ewg4F1 and ewg5R1 [56]. Amplification was done in a Applied Biosystems ABI Prism 7000 with a 3-min initial denaturation at $95^{\circ} \mathrm{C}$ followed by 40 cycles with a 15 -s denaturation at $95^{\circ} \mathrm{C}$ and $60 \mathrm{~s}$ extension at $60^{\circ} \mathrm{C}$. Quantification was done according to the $\triangle \mathrm{CT}$ method as described [44].

Structural analysis was done by PyMol. Expression data were retrieved from FlyBase (flybase.org) [35, 36]. The Nup54 ORF and extended gene sequences were retrieved from FlyBase (flybase.org) and aligned using muscle within MEGA [57]. D. melanogaster polymorphism data was retrieved from the Drosophila Genetic Reference Panel (http://dgrp2.gnets.ncsu.edu/) and used along with interspecies divergence data $(D$. melanogaster - D. simulans) to conduct McDonald Kreitman's tests of selection for the ORF and extended gene region. Tests were conducted by comparing nonsynonymous to synonymous substitutions and polymorphisms (ORF) as well as noncoding upstream to silent (within gene synonymous and intron) substitutions and polymorphism [58].

We also tested for evidence of nonrandom accumulation of substitutions along the Nup54 extended gene region. The method tests for significant deviations from a uniform distribution of substitutions using an empirical cumulative distribution function. The function $(\mathrm{G}) \mathrm{de}-$ tects monotonic increases in substitutions $(\mathrm{n})$ measured as the difference between the relative occurrence of a 
nucleotide change and its relative position in the alignment [59]. Whether differences between the values of the $\mathrm{G}$ function $(\Delta G)$ between substitutional events deviates from a random accumulation of changes is tested using Monte Carlo simulations to produce 100,000 samples of $n$ events by sampling sites without replacement along the alignment [59].

\section{Abbreviations}

Nup: Nuclear pore protein; SP: Sex-peptide; NPC: Nuclear pore complex; RNAi: Ribonucleic acid interference; SPR: Sex-peptide receptor; ppk: Pickpocket; fru: Fruitless; dsx: Doublesex; GFP: Green fluorescent protein; ORF: Open reading frame; TNT: Tetanus toxin; ELAV: Embryonic lethal abnormal visual system

\section{Supplementary Information}

The online version contains supplementary material available at https://doi. org/10.1186/s12915-021-01154-6

Additional file 1 Nup54 is required before neuronal maturation for establishing the post-mating response. A) Receptivity of control (white for Ringer and black for SP injection) and transheterozygous

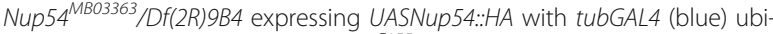
quitously, in neurons with elavGAL4 ${ }^{C 155}$ (purple) or glia with repoGAL4 (yellow), and in ppkGAL4 (orange), fruGAL4 (green) and $d s \times G A L 4$ (red) patterns after sex-peptide (SP, dark color) or Ringer's ( $R$, light color) injection measured by counting mating females in a $1 \mathrm{~h}$ time period $3 \mathrm{~h}$ after SP or $\mathrm{R}$ injection, respectively. Means with the standard error for three experiments with 15-21 females each are shown, and statistically significant differences from ANOVA post-hoc pairwise comparisons are indicated by different letters ( $p \leq 0.05)$. B) Oviposition of control (white for Ringer and black for SP injection) and transheterozygous Nup54 $4^{M B 03363} / \mathrm{Df}(2 R) 9 B 4$ expressing UASNup54::HA with tubGAL4 (blue) ubiquitously, in neurons with elavGAL4 ${ }^{C 155}$ (purple) or glia with repoGAL4 (yellow), and in ppkGAL4 (orange), fruGAL4 (green) and dsXGAL4 (red) patterns after sex-peptide (SP, dark color) or Ringer's ( $R$, light color) injection shown as means of eggs laid in $18 \mathrm{~h}$ with the standard error for 10-15 females each, respectively, and statistically significant differences from ANOVA post-hoc pairwise comparisons are indicated by different letters ( $a, b: p \leq 0.001, c: n s)$. The data underlying the presented graphs are in Additional file 6.

Additional file $\mathbf{2}$ Gross organization of the brain and ventral nerve cord is normal in Nup54 mutants. A-F) Projections of $d s x$ neurons in the brain ( $A$ and $D$ ) and ventral nerve cord (VNC, B and $E$ ) and $d s x$ expression in the genital tract ( $C$ and $F$ ) visualized by expression of membrane-bound CD8GFP from UAS by $d s \times G A L 4$ show no gross alterations in $D f(2 R) 9 B 4$ gNup54 ${ }^{\mathrm{QB} 62}$ (D-E) compared to wild type (A-C). st: spermathecae, sr: seminal receptaculum. The scale bar in D is $100 \mu \mathrm{m}$.

Additional file 3 Nup54 RNAi in doublesex expressing neurons reveals a separable sex-peptide response in receptivity and oviposition and a role in sexual differentiation. A) Receptivity after Nup54 RNAi knock-down from UAS P\{GD14041\}V42153; P\{GD14041\}V42154 inserts in neurons with elavGAL4 ${ }^{C 155}$ (yellow) and in ppkGAL4 (orange), fruGAL4 (green) and dsXGAL4 (red) patterns after sex-peptide (SP, dark color) or Ringer's ( $\mathrm{R}$, light color) injection measured by counting mating females in a $1 \mathrm{~h}$ time period $3 \mathrm{~h}$ after SP or R injection, respectively. Means with the standard error for three experiments with 16 females each are shown, and statistically significant differences from ANOVA post-hoc pairwise comparisons are indicated by different letters ( $p \leq 0.001)$. B) Oviposition after Nup54 RNAi knock-down from UAS P\{GD14041\}V42153: P\{GD14041\}v42154 inserts in neurons with elavGAL4 ${ }^{\text {C155 }}$ (yellow) and in ppkGAL4 (orange), fruGAL4 (green) and $d s x G A L 4$ (red) patterns after sex-peptide (SP, dark color) or Ringer's ( $R$, light color) injection shown as means of eggs laid in $18 \mathrm{~h}$ with the standard error for 8 females each, respectively, and statistically significant differences from ANOVA post-hoc pairwise comparisons are indicated by different letters ( $p \leq 0.0001)$. C, D) Genitals of control females (C) and females expressing Nup62 RNAi from UAS with dsXGAL4 (D). The scale bar in C is $20 \mu \mathrm{m}$. E, F) Front legs of control males (E) and males expressing Nup62 RNAi from UAS with dsXGAL4 (F). Arrowheads indicate the position of sex combs. The scale bar in $\mathrm{E}$ is $100 \mu \mathrm{m}$. The data underlying the presented graphs are in Additional file 6.

Additional file 4 Expression of Nup54, Nup58 and Nup62. A-C) Profile of Nup54, Nup58 and Nup62 expression during development from RNAseq summarized from flybase. D-F) Profile of Nup54, Nup58 and Nup62 expression in various tissues from microarrays summarized from flybase. The data underlying the presented graphs are in Additional file 6.

Additional file 5 NUP54 is highly conserved, but variation in the FG repeat region is not the cause of an altered post-mating response. A) Sequence alignment of NUP54 from closely related species. Amino acids deviating from $D$. melanogaster are indicated in black. Intron positions are indicated by black arrowheads and the stop codon of the Nup54 ${ }^{\text {MBO3363 }}$ allele is indicated by a red arrow head. Green and white filled arrowheads indicate maintained $(>1 \%)$ and rare $(<1 \%)$ polymorphisms with amino acid changes indicated on top. The line below the sequence indicates the NgoMIV-BamHI fragment that was replaced in the gNup54elegans construct. Nucleotides 1-115 according to human NUP54 impasse the FG region, nucleotides $116-346$ the $\alpha / \beta$ region and nucleotides $346-494$ the a-helical region. The amino acids in exon 3 have been shown to bind to NUP62 and the amino acids in exon 5 bind to NUP58. B) Receptivity of wild type, gNup54 and gNup54elegans females homozygous for Df(2R)9B4 after sex-peptide (SP) or Ringer's (R) injection measured by counting mating females in a $1 \mathrm{hr}$ time period $3 \mathrm{hr}$ after SP or $\mathrm{R}$ injection, respectively. Means with the standard error for three experiments with 8-15 females each are shown, and statistically significant differences from ANOVA posthoc pairwise comparisons are indicated by different letters $(p \leq 0.001)$. C) Oviposition of gNup54 and gNup54elegans females homozygous for Df(2R)9B4 after sex-peptide (SP) or Ringer's (R) injection shown as means of eggs laid in $18 \mathrm{~h}$ with the standard error for 10 females each, respectively. Statistically significant differences from ANOVA post-hoc pairwise comparisons are indicated by different letters ( $p \leq 0.001)$. The data underlying the presented graphs are in Additional file 6.

Additional file 6. Source data underlying the presented graphs.

\section{Acknowledgements}

We thank T. Schüpbach, B. Dickson, S. Goodwin, C. Rezaval, D. Anderson, S. Collier, the Bloomington Stock Center, the Vienna Drosophila RNAi Center, and the Arizona Species Stock Center for fly lines and the University of Cambridge Department of Genetics Fly Facility for injections; N. Arora for help with behavioral assays; D. Scocchia for help with graphics and qPCR; T. Dix for help with PyMol; and C. Rezaval and J.C. Billeter for comments on the manuscript. We are indebted to Eric Kubli for his support when this study was initiated.

\section{Authors' contributions}

MS conceived and directed the project. IUH and MS performed the molecular biology experiments; IUH, MN, and MS performed the genetic experiments; and AC performed the sequence analysis. All authors analyzed the data. MS wrote the manuscript with support from IUH and AC. The authors read and approved the final manuscript.

\section{Authors' Information}

Twitter handle: Mohanakarthik P. Nallasivan, @MohanakarthikPN; Irmgard U. Haussmann, @lrmgardHaussman; Matthias Soller, @MatthiasSoller.

\section{Funding}

This work was supported by the Biotechnology and Biological Science Research Council to MS and the Natural Sciences and Engineering Research Council of Canada to AC.

\section{Availability of data and materials}

All data generated or analyzed during this study are included in this published article and its supplementary information files. Reagents are available from the corresponding author upon request. 


\section{Declarations}

\section{Ethics approval and consent to participate}

Not applicable.

\section{Consent for publication}

Not applicable.

\section{Competing interests}

The authors declare no competing interests.

\section{Author details}

${ }^{1}$ School of Biosciences, College of Life and Environmental Sciences, University of Birmingham, Edgbaston, Birmingham B15 2TT, UK. ${ }^{2}$ Department of Life Science, School of Health Sciences, Birmingham City University, Birmingham B15 3TN, UK. ${ }^{3}$ Department of Biology, University of Winnipeg, Winnipeg, MB R3B 2E9, Canada. ${ }^{4}$ Birmingham Centre for Genome Biology, University of Birmingham, Edgbaston, Birmingham B15 2TT, UK.

\section{Received: 18 March 2021 Accepted: 15 September 2021}

\section{Published online: 20 October 2021}

\section{References}

1. Gillott C. Male accessory gland secretions: Modulators of Female Reproductive Physiology and Behavior. Annu Rev Entomol. 2003;48(1):16384. https://doi.org/10.1146/annurev.ento.48.091801.112657.

2. Avila FW, Sirot LK, LaFlamme BA, Rubinstein CD, Wolfner MF. Insect seminal fluid proteins: identification and function. Annu Rev Entomol. 2011;56(1):2140. https://doi.org/10.1146/annurev-ento-120709-144823.

3. Kubli E. Sex-peptides: seminal peptides of the Drosophila male. Cell Mol Life Sci. 2003:60(8):1689-704. https://doi.org/10.1007/s00018-003-3052.

4. Soller M, Bownes M, Kubli E. Control of oocyte maturation in sexually mature Drosophila females. Dev Biol. 1999;208(2):337-51. https://doi.org/1 0.1006/dbio.1999.9210

5. Carvalho GB, Kapahi P, Anderson DJ, Benzer S. Allocrine modulation of feeding behavior by the Sex Peptide of Drosophila. Curr Biol. 2006;16(7): 692-6. https://doi.org/10.1016/j.cub.2006.02.064.

6. Isaac RE, Li C, Leedale AE, Shirras AD. Drosophila male sex peptide inhibits siesta sleep and promotes locomotor activity in the post-mated female. Proc Biol Sci. 2010;277(1678):65-70. https://doi.org/10.1098/rspb.2009.1236.

7. Ribeiro C, Dickson BJ. Sex peptide receptor and neuronal TOR/S6K signaling modulate nutrient balancing in Drosophila. Curr Biol. 2010;20(11):1000-5. https://doi.org/10.1016/j.cub.2010.03.061.

8. Peng J, Zipperlen P, Kubli E. Drosophila sex-peptide stimulates female innate immune system after mating via the Toll and Imd pathways. Curr Biol. 2005;15(18):1690-4. https://doi.org/10.1016/j.cub.2005.08.048.

9. Domanitskaya EV, Liu H, Chen S, Kubli E. The hydroxyproline motif of male sex peptide elicits the innate immune response in Drosophila females. FEBS J. 2007;274(21):5659-68. https://doi.org/10.1111/j.1742-4658.2007.06088.x.

10. Cognigni P, Bailey AP, Miguel-Aliaga I. Enteric neurons and systemic signals couple nutritional and reproductive status with intestinal homeostasis. Cell Metab. 2011:13(1):92-104. https://doi.org/10.1016/j.cmet.2010.12.010.

11. Scheunemann L, Lampin-Saint-Amaux A, Schor J, Preat T. A sperm peptide enhances long-term memory in female Drosophila. Sci Adv. 2019;5:eaax3432.

12. Peng J, Chen S, Busser S, Liu H, Honegger T, Kubli E. Gradual release of sperm bound sex-peptide controls female postmating behavior in Drosophila. Curr Biol. 2005;15(3):207-13. https://doi.org/10.1016/j.cub.2005.01.034

13. Avila FW, Ravi Ram K, Bloch Qazi MC, Wolfner MF. Sex peptide is required for the efficient release of stored sperm in mated Drosophila females. Genetics. 2010;186(2):595-600. https://doi.org/10.1534/genetics.110.119735.

14. Wigby S, Chapman T. Sex peptide causes mating costs in female Drosophila melanogaster. Curr Biol. 2005;15(4):316-21. https://doi.org/10.1016/j.cub.2 005.01 .051 .

15. Soller M, Haussmann IU, Hollmann M, Choffat $Y$, White K, Kubli E, et al. Sexpeptide-regulated female sexual behavior requires a subset of ascending ventral nerve cord neurons. Curr Biol. 2006;16(18):1771-82. https://doi.org/1 0.1016/j.cub.2006.07.055

16. Fan Y, Soller M, Flister S, Hollmann M, Muller M, Bello B, et al. The egghead gene is required for compartmentalization in Drosophila optic lobe development. Dev Biol. 2005;287(1):61-73. https://doi.org/10.1016/j.ydbio.2 005.08.031.
17. Hasemeyer M, Yapici N, Heberlein U, Dickson BJ. Sensory neurons in the Drosophila genital tract regulate female reproductive behavior. Neuron 2009;61(4):511-8. https://doi.org/10.1016/j.neuron.2009.01.009.

18. Yang CH, Rumpf S, Xiang Y, Gordon MD, Song W, Jan LY, et al. Control of the postmating behavioral switch in Drosophila females by internal sensory neurons. Neuron. 2009:61(4):519-26. https://doi.org/10.1016/j.neuron.2 008.12.021.

19. Rezaval C, Pavlou HJ, Dornan AJ, Chan YB, Kravitz EA, Goodwin SF. Neural circuitry underlying Drosophila female postmating behavioral responses. Curr Biol. 2012;22(13):1155-65. https://doi.org/10.1016/j.cub.2012.04.062.

20. Yapici N, Kim YJ, Ribeiro C, Dickson BJ. A receptor that mediates the postmating switch in Drosophila reproductive behaviour. Nature. 2008; 451(7174):33-7. https://doi.org/10.1038/nature06483.

21. Haussmann IU, Hemani Y, Wijesekera T, Dauwalder B, Soller M. Multiple pathways mediate the sex-peptide-regulated switch in female Drosophila reproductive behaviours. Proc Biol Sci. 2013;280(1771):20131938. https://doi. org/10.1098/rspb.2013.1938.

22. Sirot LK, Wong A, Chapman T, Wolfner MF. Sexual conflict and seminal fluid proteins: a dynamic landscape of sexual interactions. Cold Spring Harb Perspect Biol. 2014;7(2):a017533. https://doi.org/10.1101/cshperspect.a017533.

23. Chapman T. Sexual conflict: mechanisms and emerging themes in resistance biology. Am Nat. 2018;192(2):217-29. https://doi.org/10.1086/698169.

24. Hollis B, Koppik M, Wensing KU, Ruhmann H, Genzoni E, Erkosar B, et al. Sexual conflict drives male manipulation of female postmating responses in Drosophila melanogaster. Proc Natl Acad Sci U S A. 2019;116(17):8437-44. https://doi.org/10.1073/pnas.1821386116.

25. Presgraves DC, Balagopalan L, Abmayr SM, Orr HA. Adaptive evolution drives divergence of a hybrid inviability gene between two species of Drosophila. Nature. 2003;423(6941):715-9. https://doi.org/10.1038/nature01679.

26. Tang S, Presgraves DC. Evolution of the Drosophila nuclear pore complex results in multiple hybrid incompatibilities. Science. 2009;323(5915):779-82. https://doi.org/10.1126/science.1169123.

27. Presgraves DC. The molecular evolutionary basis of species formation. Nat Rev Genet. 2010;11(3):175-80. https://doi.org/10.1038/nrg2718.

28. Sawamura K, Maehara K, Mashino S, Kagesawa T, Kajiwara M, Matsuno K, et al. Introgression of Drosophila simulans nuclear pore protein 160 in Drosophila melanogaster alone does not cause inviability but does cause female sterility. Genetics. 2010;186(2):669-76. https://doi.org/10.1534/ genetics.110.119867.

29. Lin $\mathrm{DH}$, Hoelz $\mathrm{A}$. The structure of the nuclear pore complex (an update). Annu Rev Biochem. 2019;88(1):18.11-59. https://doi.org/10.1146/annurevbiochem-062917-011901.

30. Schupbach T, Wieschaus E. Female sterile mutations on the second chromosome of Drosophila melanogaster. II. Mutations blocking oogenesis or altering egg morphology. Genetics. 1991;129(4):1119-36. https://doi.org/1 0.1093/genetics/129.4.1119.

31. Haussmann IU, White $K$, Soller M. Erect wing regulates synaptic growth in Drosophila by integration of multiple signaling pathways. Genome Biol. 2008;9(4):R73. https://doi.org/10.1186/gb-2008-9-4-r73.

32. Zaharieva E, Haussmann IU, Brauer U, Soller M. Concentration and localization of co-expressed ELAV/Hu proteins control specificity of mRNA processing. Mol Cell Biol. 2015;35(18):3104-15. https://doi.org/10.1128/MCB.00473-15.

33. Lamaze A, Ozturk-Colak A, Fischer R, Peschel N, Koh K, Jepson JE. Regulation of sleep plasticity by a thermo-sensitive circuit in Drosophila. Sci Rep. 2017; 7(1):40304. https://doi.org/10.1038/srep40304.

34. Asahina K, Watanabe K, Duistermars BJ, Hoopfer E, Gonzalez CR, Eyjolfsdottir EA, et al. Tachykinin-expressing neurons control male-specific aggressive arousal in Drosophila. Cell. 2014;156(1-2):221-35. https://doi.org/10.1016/j. cell.2013.11.045

35. Chintapalli VR, Wang J, Dow JA. Using FlyAtlas to identify better Drosophila melanogaster models of human disease. Nat Genet. 2007;39(6):715-20. https://doi.org/10.1038/ng2049.

36. Brown JB, Boley N, Eisman R, May GE, Stoiber MH, Duff MO, et al. Diversity and dynamics of the Drosophila transcriptome. Nature. 2014;512(7515):3939. https://doi.org/10.1038/nature12962.

37. Barton HJ, Zeng K. The impact of natural selection on short insertion and deletion variation in the great tit genome. Genome Biol Evol. 2019;11(6): 1514-24. https://doi.org/10.1093/gbe/evz068.

38. Raices M, D'Angelo MA. Nuclear pore complex composition: a new regulator of tissue-specific and developmental functions. Nat Rev Mol Cell Biol. 2012;13(11):687-99. https://doi.org/10.1038/nrm3461. 
39. Kim HJ, Taylor JP. Lost in transportation: nucleocytoplasmic transport defects in ALS and other neurodegenerative diseases. Neuron. 2017;96(2): 285-97. https://doi.org/10.1016/j.neuron.2017.07.029.

40. Solomon DA, Stepto A, Au WH, Adachi Y, Diaper DC, Hall R, et al. A feedback loop between dipeptide-repeat protein, TDP-43 and karyopherinalpha mediates C9orf72-related neurodegeneration. Brain. 2018;141(10): 2908-24. https://doi.org/10.1093/brain/awy241.

41. D'Angelo MA, Gomez-Cavazos JS, Mei A, Lackner DH, Hetzer MW. A change in nuclear pore complex composition regulates cell differentiation. Dev Cell. 2012;22(2):446-58. https://doi.org/10.1016/j.devcel.2011.11.021.

42. Jacinto FV, Benner C, Hetzer MW. The nucleoporin Nup153 regulates embryonic stem cell pluripotency through gene silencing. Genes Dev. 2015; 29(12):1224-38. https://doi.org/10.1101/gad.260919.115.

43. Hazawa M, Lin DC, Kobayashi A, Jiang YY, Xu L, Dewi FRP, et al. ROCKdependent phosphorylation of NUP62 regulates p63 nuclear transport and squamous cell carcinoma proliferation. EMBO Rep. 2018;19(1):73-88. https:// doi.org/10.15252/embr.201744523.

44. Haussmann IU, Bodi Z, Sanchez-Moran E, Mongan NP, Archer N, Fray RG, et al. m6A potentiates Sxl alternative pre-mRNA splicing for robust Drosophila sex determination. Nature. 2016;540(7632):301-4. https://doi. org/10.1038/nature20577.

45. Schutt C, Nothiger R. Structure, function and evolution of sex-determining systems in Dipteran insects. Development. 2000;127(4):667-77. https://doi. org/10.1242/dev.127.4.667.

46. Munafo M, Lawless VR, Passera A, MacMillan S, Bornelov S, Haussmann IU, et al. Channel nuclear pore complex subunits are required for transposon silencing in Drosophila. Elife. 2021;10:e66321. https://doi.org/10.7554/eLife. 66321.

47. Graindorge A, Carre C, Gebauer F. Sex-lethal promotes nuclear retention of msl2 mRNA via interactions with the STAR protein HOW. Genes Dev. 2013; 27(12):1421-33. https://doi.org/10.1101/gad.214999.113

48. Kelleher ES, Edelman NB, Barbash DA. Drosophila interspecific hybrids phenocopy piRNA-pathway mutants. PLoS Biol. 2012;10(11):e1001428. https://doi.org/10.1371/journal.pbio.1001428.

49. Parhad SS, Tu S, Weng Z, Theurkauf WE. Adaptive evolution leads to cross-species incompatibility in the piRNA transposon silencing machinery. Dev Cell. 2017;43(1):60-70 e65. https://doi.org/10.1016/j. devcel.2017.08.012.

50. Czech B, Preall JB, McGinn J, Hannon GJ. A transcriptome-wide RNAi screen in the Drosophila ovary reveals factors of the germline piRNA pathway. Mol Cell. 2013;50(5):749-61. https://doi.org/10.1016/j.molcel.2013.04.007.

51. Hirai K, Wang Z, Miura K, Hayashi T, Awasaki T, Wada M, et al. Genetic analyses of elys mutations in Drosophila show maternal-effect lethality and interactions with nucleoporin genes. G3. 2018;8:2421-31.

52. Ilyin AA, Ryazansky SS, Doronin SA, Olenkina OM, Mikhaleva EA, Yakushev EY, et al. Piwi interacts with chromatin at nuclear pores and promiscuously binds nuclear transcripts in Drosophila ovarian somatic cells. Nucleic Acids Res. 2017;45(13):7666-80. https://doi.org/10.1093/nar/gkx355.

53. Lisbin MJ, Gordon M, Yannoni YM, White K. Function of RRM domains of Drosophila melanogaster ELAV: RNP1 mutations and RRM domain replacements with ELAV family proteins and SXL. Genetics. 2000;155(4): 1789-98. https://doi.org/10.1093/genetics/155.4.1789.

54. Haussmann IU, Ustaoglu P, Brauer U, Hemani Y, Dix TC, Soller M. Plasmidbased gap-repair recombineered transgenes reveal a central role for introns in mutually exclusive alternative splicing in Down Syndrome Cell Adhesion Molecule exon 4. Nucleic Acids Res. 2019;47(3):1389-403. https://doi.org/1 $0.1093 /$ nar/gky 1254

55. Koushika SP, Soller M, DeSimone SM, Daub DM, White K. Differential and inefficient splicing of a broadly expressed Drosophila erect wing transcript results in tissue-specific enrichment of the vital EWG protein isoform. Mol Cell Biol. 1999;19(6):3998-4007. https://doi.org/10.1128/MCB.19.6.3998.

56. Haussmann IU, Li M, Soller M. ELAV-mediated 3'-end processing of ewg transcripts is evolutionarily conserved despite sequence degeneration of the ELAV-binding site. Genetics. 2011;189(1):97-107. https://doi.org/10.1534/ genetics.111.131383.

57. Edgar RC. MUSCLE: multiple sequence alignment with high accuracy and high throughput. Nucleic Acids Res. 2004;32(5):1792-7. https://doi.org/10.1 093/nar/gkh340
58. Huang W, Massouras A, Inoue Y, Peiffer J, Ramia M, Tarone AM, et al. Natural variation in genome architecture among 205 Drosophila melanogaster Genetic Reference Panel lines. Genome Res. 2014;24(7):1193-208. https:// doi.org/10.1101/gr.171546.113

59. Civetta A, Ostapchuk DC, Nwali B. Genome hotspots for nucleotide substitutions and the evolution of influenza $A(\mathrm{H} 1 \mathrm{~N} 1)$ human strains. Genome Biol Evol. 2016;8(4):986-93. https://doi.org/10.1093/gbe/evw061.

\section{Publisher's Note}

Springer Nature remains neutral with regard to jurisdictional claims in published maps and institutional affiliations.
Ready to submit your research? Choose BMC and benefit from:

- fast, convenient online submission

- thorough peer review by experienced researchers in your field

- rapid publication on acceptance

- support for research data, including large and complex data types

- gold Open Access which fosters wider collaboration and increased citations

- maximum visibility for your research: over $100 \mathrm{M}$ website views per year

At BMC, research is always in progress.

Learn more biomedcentral.com/submissions 